\title{
High Speed CCD Photometry of Flare Stars
}

\author{
Sun-Youp Park \\ Department of Astronomy, Yonsei University, Seoul, 120-749, Korea \\ Yong-Ik Byun \\ Department of Astronomy, Yonsei University, Seoul, 120-749, Korea
}

\begin{abstract}
Previously, flare stars have been observed by fast two channel photoelectric photometry. We have started an observing program to monitor flare stars using a normal CCD camera via a method called "trailed mode photometry". For this program, we developed a fully automated trail photometry software.
\end{abstract}

\section{Introduction}

Solar and stellar flares are believed to take place because of the heating of the corona by X-ray emission and material evaporation from dense heated region of the chromosphere. There are however several different models for solar and stellar flare activities. While flares in general share some common features, they can be radically different in others. For example, the total energy released during a stellar flare of dMe-type flare stars can be up to 1,000 times greater than that of the Sun. More observations are needed to understand the variety and nature of flare events. Previously, flare stars have been observed by fast two channel photoelectric photometry; typical timescales of flare phenomena are $1 \sim 100$ seconds. We have started an observing program to monitor flare stars using a normal CCD camera via a method called "trailed mode photometry". For this program, we developed a fully automated trail photometry software. Our study will later be expanded into a general survey to search for unknown flare star population.

We describe here the details of our photometry software algorithm.

\section{Outline of Photometry Procedure}

The steps for our photometry can be outlined as follows. First, we obtain an image, as shown at the right of Figure 1 with a normal CCD camera; this is a "trail image" taken with the telescope tracking at a speed slower than siderial rate. After the basic pre-processing (bias, dark, flat field, bad pixel \& cosmic ray removal), local peaks are detected using an algorithm similar to DAOPHOT. Then, by continuation of detected peaks, the locations of each trail in the image are defined. Finally, the light variations of each object along the trails are extracted. 

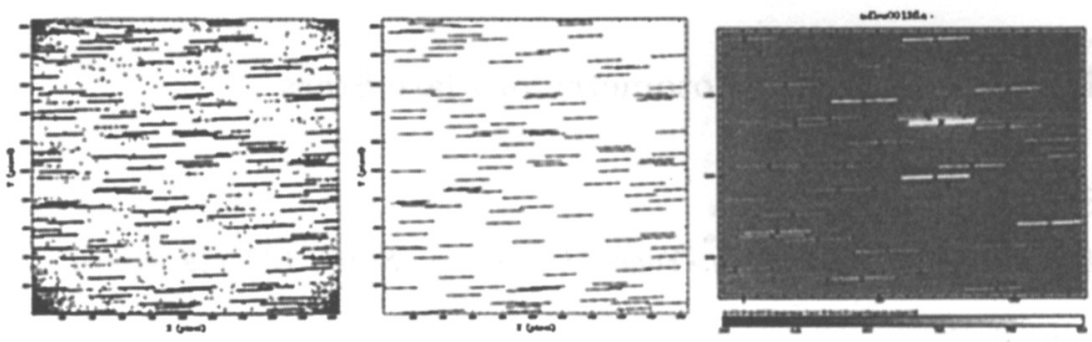

Figure 1. Steps for detection trails. Left : peak detection. Middle : Peak continuation. Right : Trail locations marked on the trail image.
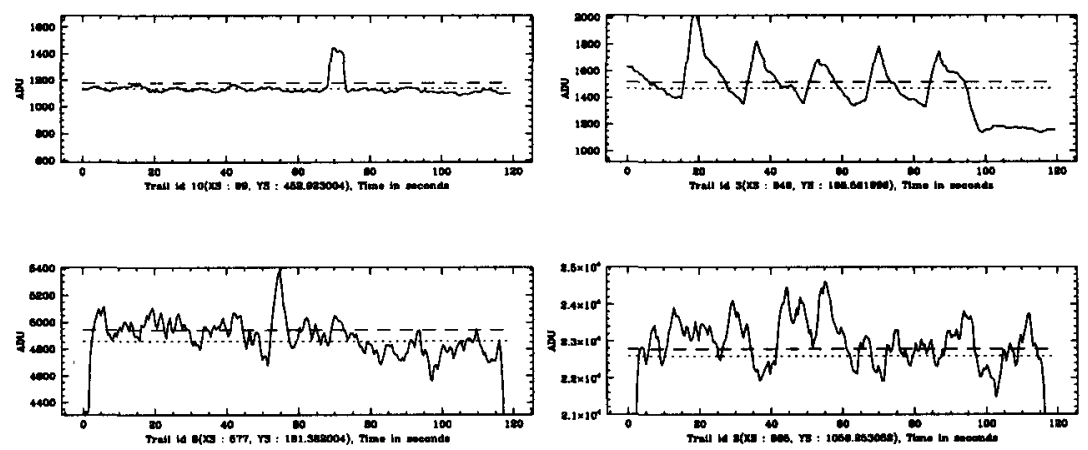

Figure 2. Trail light curves of some fast varying objects. Flux variations (in ADU) versus time (in seconds) are shown. Dotted lines and dashed lines show the average and the 3- $\sigma$ threshold levels, respectively.

\section{Discussions}

Our study can be summarized as follows. First, although CCDs are slow-readout devices, it is possible to study fast-varying objects using trailed mode photometry. Second, with automation, it is possible to extract and examine light curves for a large number of objects with a single trail image. Third, the application of this kind of photometry includes, but is not limited to, the study of flare stars, Gamma-ray Bursts, and other fast transient events. This method is however limited by fast variations caused by the earth's atmosphere, such as scintillation, which needs further study. 